\title{
Anti-inflammatory Mechanism of Rhein in Treating Asthma Based on Network Pharmacology
}

\section{Junfang Feng}

Shandong University

Ou Chen

Shandong University of Nursing School

Yibiao Wang ( $\square$ wangyibiao@sdu.edu.cn )

Shandong University

\section{Research}

Keywords: Asthma, Rhein, Anti-inflammatory, Network pharmacology, Rhein-Predicted target, pathway

Posted Date: January 29th, 2020

DOI: https://doi.org/10.21203/rs.2.22156/v1

License: (1) This work is licensed under a Creative Commons Attribution 4.0 International License. Read Full License 


\section{Abstract}

\section{Background}

Network pharmacology was used to study Rhein -target-pathway and to clarify its anti-inflammatory mechanism in the treatment of asthma.and provide a new idea for the treatment of asthma

\section{Methods}

This method, which allows using network pharmacology to figure out the operational mechanism of Rhein-Target-Pathway, defines the effect of anti-inflammatory in treating asthma. The platform of Traditional Chinese Medicine Molecular Mechanism Bioinformatics, a web server for network, is used to get the corresponding target of Rhein and permit molecular docking. Cytoscape3.7.1, a kind of network software, is used to construct Rhein-predicted target network and analyse network topology. Search antiinflammatory targets in the database of TTD and then construct the PPI network as well as create protein interaction networks that are combined with the Rhein-predicted target network. The anti-inflammatory targets of Rhein should be presented. The asthma genes of human being can be attained from the database of NCBI Gene Database and construct correspondence vivo response network model. Find Antiinflammatory targets of Rhein against asthma, screen anti-inflammatory targets of Rhein related with Pathogenesis of asthma. Enrichr database is used to analyse signal pathway from anti-inflammatory targets of Rhein KEGG.

\section{Results}

According to the study, Rhein corresponds to 17 target proteins, four anti-inflammatory targets of Rhein related to asthma(MAPK14, EGFR, ERBB2, TNFRSF1A) are probably the most important targets where asthma is treated by Rhein.

\section{Conclusions}

These four anti-inflammatory targets of Rhein related to asthma are probably the key targets in the treatment of asthma by using Rhein. For the purpose of preventing the occurrence as well as development of asthma and delaying the progress of the disease, one or some of the four antiinflammatory targets of Rhein related to asthma can be controlled.

\section{Background}

Asthma is abbreviated for Bronchial Asthma. It is a kind of chronic inflammatory disorder of the airways involved of cells and corresponding components, which is characterized by inflammation, hyper responsiveness, stenosis and remodelling of the airway. The chronic inflammation is considered as the hypostasis of asthma [1, 2]. 
Rhein is one of the effective monomer components that are isolated and then purified from rhubarb which belongs to Chinese herbs. Rhein, which is a monanthraquinone 1, 8-dihydroxyl anthraquinone derivative, permits anti-inflammatory, anti-bacterial, anti-tumour and other effect $[3,4,5,6]$. As far as it goes, we have probably not found any report that Rhein can be used to treating asthma by its antiinflammatory.

Network pharmacology, which is an emerging research method for the past few years, is considered to be a new model for the next generation of drug research. While the construction of biomolecular networks such as "drug-target-path" are the basis of network pharmacolog $[7,8]$.

We can explore the efficacy of drugs from the component-target-pathway, and can also reverse the pathogenesis of the disease through drugs with known therapeutic effect $[9,10]$. Hence, by exploring the anti-inflammatory mechanism of rhubarb acid in treating asthma with network pharmacology method, a newer and more advanced view may be provided to treat asthma.

\section{Materials And Methods}

\section{Tools used and data sources}

Search for chemical composition of the drug and its targets on the web of TCMSP, PubChem, TTD, STITCH, Drugbank Database; It is possible to get the genes of related diseases and the Information on gene interactions and protein-protein interactions from Genebank Database, String Database; Signal pathways including biomolecules could be found by searching Biocarta and KEGG; Cytoscape 3.7.1 and Systemsdock are respectively for network construction and molecular docking.

\section{Prediction of the target of Rhein prediction}

Three-dimensional chemical structure data of Rhein, where it can be searched and exported from TCMSP and PubChem, is imported into the database of Swiss prediction target and do the reverse molecular docking. Predictive target can be gotten by setting the target set "homo sapiens". The result can be used for further study.

\section{Molecular docking to Rhein-Target protein}

The PDB-ID in PDB Database is imported into the Systemsdock where the molecular docking can be processed. The value of the Docking Score is used to judge the matching degree between rhubarb acid and target. The score ranges from $0-10$, the greater the value, the more stable the ligand binds to the receptor [11].

\section{Construction of Rhein-Target Network}

Construct a 'drug-target' interaction network with Rhein and its potential targets by using the software Cytoscape. 


\section{Construction of Rhein-Target Protein Interaction Network}

By using STRING, construct Rhein-Target Protein Interaction Network by setting Protein type into Homo sapiens, the minimum interaction threshold is set to medium "medium confidence" and the remaining parameters are kept silent.

\section{Anti-inflammatory related target protein screening}

In the database TTD, search for information of anti-inflammatory target protein by using the keyword "anti-inflammation" which is put into Cytoscape3.7.1 to construct anti-inflammatory target protein PPI network.

\section{Screening of anti-inflammatory targets for Rhein-effect and network construction of anti-inflammatory targets}

Combine the Rhein-Target Protein Network with the Anti-inflammatory Protein PPI Network and import the result, which is called anti-inflammatory targets for Rhein-effect, into the database of String and construct the network. Screen values which are beyond 0.7 as high confidence basis for protein interactions.

\section{Search for asthma related genes of human being}

In NCBI Gene Database (http://www.ncbi.nim.nih.gov), 'asthma' and 'homo sapiens' are searchable keywords, the asthma-related genes of a human being can be acquired.

\section{Network of the anti-inflammatory target of Rhein against asthma}

Import the anti-inflammatory target genes of Rhein and human asthma-related genes into the String database to construct the response network of drug anti-inflammatory targets against asthma in vivo and screen the anti-inflammatory targets related to asthma incidence.

\section{Enrichment pathways of the target genes to KEGG}

Use the Enrichr database to analyse the biological enrichment pathways of the target genes to KEGG for the prediction of rhein anti-inflammatory targets.

\section{Results}

The reverse docking score of Rhein and related targets, which are all beyond 5 , verifying the reliability of the predicted target (Tab. 1).

\section{Rhein-Anti-inflammatory target PPI network analysis}

Nine anti-inflammatory proteins are detected in TTD, and in PPI network (Fig.3), there were nine interacting targets, constituting seven interacting relationships. 
The Cytoscape 3.7.1 merge function is used to fuse the Rhein-Predicted target network to the antiinflammatory target PPI network in the TTD, taking the intersection (Fig. 4), taking a high confidence interval beyond 0.7 to obtain Rhein resistance inflammatory targets, including mitogen-activated protein kinase (MAPK14), receptor tyrosine-protein kinase erbB-2 (ERBB2), tumor necrosis factor receptor superfamily member 1A (TNFRSF1A), epidermal growth factor receptor (EGFR).

\section{Reaction network of Rhein anti-inflammatory targets against asthma (Fig. 5)}

The Cytoscape 3.7.1 merge function was used to fuse the Rhein-predicted target network to the antiinflammatory target PPI network in the TTD, taking the intersection (Fig. 5), taking a high confidence interval greater than 0.7 to obtain rhein resistance inflammatory targets, including mitogen-activated protein kinase (MAPK14), receptor tyrosine-protein kinase erbB-2 (ERBB2), tumor necrosis factor receptor superfamily member 1A (TNFRSF1A), epidermal growth factor receptor (EGFR).

\section{Enrichment pathways of the target genes to KEGG}

According to enrichment pathways of the target genes to KEGG, anti-inflammatory target protein of Rhein associated with asthma involves 87 signalling pathways. Enrichr analysis results are sorted in the descending order of Combined Score. The top ten pathways contain MAPK signalling pathway Hepatitis $\mathrm{C} \bigotimes$ Epithelial cell signalling in Helicobacter pylori infection, immune signalling pathway, and cancer signalling pathway proteoglycans in cancer, Bladder cancer, Non-small cell lung cancer, Endometrial cancer, Pancreatic cancer, Central carbon metabolism in cancer, Amyotrophic lateral sclerosis (ALS) (Fig. $6)$.

\section{Discussion}

It has been reported that Rhein has anti-inflammatory activity $[12,13]$. The results of this study show the anti-inflammatory effects of Rhein. Youdong Xu et al. explained the anti-inflammatory effects of Rhein

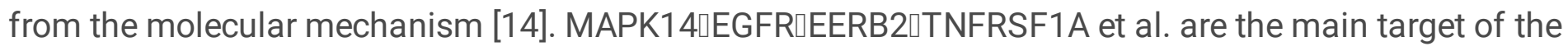

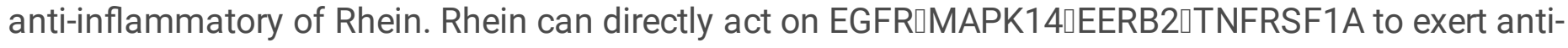
inflammatory effects, and can also act indirectly on other targets to exert its anti-inflammatory effects[15].

EGFR is an epidermal growth factor receptor, which is widely distributed in epithelial tissues and plays an important regulatory role in the development of respiratory inflammation [16]. GFR inhibitor can effectively inhibit acute inflammation of the rat respiratory tract caused by exogenous zinc ion [17]. EGFR inhibitors reduce the symptoms of allergic asthma caused by dust mites by reducing the production of pro-inflammatory factors such as IL-6 and IL-8 [18]. Thus, it is speculated that Rhein interacts with EGFR, thereby blocking the binding of EGFR to pro-inflammatory cytokines to exert an anti-inflammatory effect.

MAPK14 plays an important role in the cellular cascade triggered by pro-inflammatory cytokines or extracellular stimuli and is a key signalling molecule for lung tissue inflammation induced by $\mathrm{S}$. 
pneumonia $[19,20]$. Inflammatory factors such as IL-1 $\beta$, IL-6 and TNF-a can positively feedback the signalling pathways such as ERK and nuclear factor-KB (NF-KB) by activating the p38MAPK signalling pathway and cascading amplifying the inflammatory response [21, 22]. The transcriptional cascade regulated by P38MAPKs leads to the production of pro-inflammatory factors such as TNF- $\alpha$ and IL-1 $\beta$, which in turn leads to the activation of enzymes involved in inflammation [23]. It is speculated that Rhein exerts its anti-inflammatory effect by inhibiting the release of pro-inflammatory factors such as TNF-a and IL-1 $\beta$ by inhibiting MAPK14.

TNFRSF1A is a type 1 TNF receptor that mediates inflammatory responses mainly by activating NF-KB, p38, ERK1/2 to induce IL-6, IL-8 synthesis and apoptosis $[24,25]$. It is thus concluded that Rhein interacts with TNFRSF1A to reduce the release of anti-inflammatory factors such as IL-6 and exert an antiinflammatory effect.

EERB2, also called HER2, NEU, CD340, is a $185 \mathrm{kD}$ a cell membrane receptor encoded by the protooncogene erbB-2 and is a member of the EGFR family. EERB2 can induce IL- 6 autocrine, which in turn affects JAK-STAT or NF-KB pathway-mediated inflammatory response [26]. It is speculated that Rhein interacts with EERB2 and may exert anti-inflammatory effects by regulating the secretion of IL-6 [27, 28 , 29].

In this experiment, the anti-inflammatory mechanism of Rhein was confirmed by the method of network pharmacology. The main target of the anti-inflammatory effect of Rhein and related signalling pathways were predicted. It was found that Rhein exerted an anti-inflammatory effect by acting on multiple targets. However, network pharmacology research is based on network modelling, database resource development and software application. The network model has certain differences with the internal environment. Therefore, the research results of the anti-inflammatory effects of Rhein need to be confirmed by further experiments.

\section{Declarations}

\section{Acknowledgements}

Not applicable

\section{Funding}

No funding was received

\section{Availability of data and materials}

The datasets used and/or analysed during the current study are available from the corresponding author on reasonable request $₫$ Supplementary Table SII区.

\section{Authors' contributions}


All authors read and approved the final manuscript.

\section{Ethics approval and consent to participate}

The experimental protocol was established, according to the ethical guidelines of the Helsinki Declaration and was approved by the Human Ethics Committee. Written informed consent was obtained from individual or guardian participants.

\section{Patient consent for publication}

Not applicable

\section{Publication of clinical datasets}

Not applicable

\section{Competing interests}

The authors declare that they have no competing interests.

\section{Authors' information}

1 Author: Junfang Feng, doctoral student of School of Clinical Medicine,Shandong University, Jinan, P.R.China; currently working department of Pediatrics in Dezhou People's Hospital Pediatrics Dezhou, P.R.China. Email: dzsunshine@163.com. Address: School of Clinical Medicine, Shandong University, Jinan, P.R.China; \#44 West Wenhua Road, Jinan 250012, P.R.China.

2 Correspondence to: Ou Chen, Professor of Department of Nursing Management, Nursing School, Shandong University. Email: chenou@sdu.edu.cn. Address: Department of Nursing Management, Nursing School, Shandong University, \#44 West Wenhua Road, Jinan 250012, P.R.China.

3 Correspondence to: Yibiao Wang, Professor of Department of Pediatrics, Second Hospital, Shandong University. Email: wangyibiao@sdu.edu.cn. Address: Department of Pediatrics, Second Hospital, Shandong University, \#247 Beiyuan Road, Jinan 250033, P.R.China. Tel..+86 531 85875005; Fax: +86 531 82942003.

\section{References}

1. Alberto Papi, Christopher Brightling, et al. Asthma. Lancet. 2018; 391:783-800.

2. Samitas K, Delimpoura V, Zervas, et al. Anti-IgE treatment, airway inflammation and remodeling in severe allergic asthma: current knowledge and future perspectives. Eur Respir Rev. 2015; 24(138): 594-601.

3. Tangyi Peng, Qinjun Yang. The application of Rhein in Treatise on Cold Pathogenic and Miscellaneous Diseases. Journal of Henan Medicine. 2018; 38(3): 336-338. 
4. Weike Feng, Huayun Yu, Yuan Wang, et al. Effects of berberine and Rhein on LPS-induced macrophage inflammatory response and TLR2/NF-KB signaling pathway. Shandong Medical Journal. 2018; 58:42.

5. Qiuhe Chen, Rongbiao Pi, Jingkao Chen. Rhein and its derivatives: progress in synthesis and pharmacological research. Journal of Pharmaceutical Research. 2018; 35(3):161-167.

6. Zhuang Shen, Zhong Jia, Yifei Bian, et al: Rhein ameliorates lipopolysaccharide-induced intestinal barrier injury via modulation of Nrf2 and MAPKs. Life Sciences. 2019; 168-175.

7. Bolong Wang. Study on Active Component-Target-Pathway of Zhizhu Decoction Based on Network Pharmacology. Traditional Chinese Drugs Research and Clinical Pharmacology. 2018; 29(6):759-767.

8. Zhiqiang Liu, Bolong Wang. Research progress on screening and target prediction of network pharmacology. Traditional Chinese medicine. 2019; 41(1):171-178.

9. Yanqiong Zhang, Shao Li. Advances in network pharmacology and modern research on traditional Chinese medicine. Chinese Journal of Pharmacology and Toxicology. 2015; 29『6『: 883-891.

10. Ou Chen, Guoyong Liu, Aihong Liu, et al. Network pharmacology predicts the anti-inflammatory mechanism of ephedra in the treatment of asthma. Journal of Shandong University (Medical Edition). 2019; 57(1):55-61.

11. Kun-Yi H, Yukiko M, Yoshiyuki A, et al. Systemsdock: a web server of network pharmacology-based prediction and analysis. J Nucleic Acids Res. 2016; 44(1), 507-513.

12. Zhang $\mathrm{K}$, Jiao XF, Li JX, et al. Rhein inhibits lipopolysaccharide-induced intestinal injury during sepsis by blocking the toll-like receptor 4 nuclear factor-KB pathway. Mol Med Rep 2015; 12(3):4415-4421.

13. Fang Cao, Haitao Li, Mingming Wang. Therapeutic effect of rhein on rats with acute lung injury induced by oleic acid and its effect on pulmonary microvascular barrier and inflammatory response. Guizhou Medicine 2018; 42(8):917-921.

14. Youdong Xu, Yan Zhang, Xianli Meng. Anti-inflammatory effect of rhein based on reverse docking molecular mechanism research. Sichuan Journal of Physiological Sciences. 2016 38(1) :1-4.

15. Qilin Lei, Yalan Huang, Qian Zhong. Study on anti-inflammatory mechanism of Astragalus membranaceus based on network pharmacology. Journal of Chinese herbal medicine. 2018; 49(15):3523-3530.

16. Zhang $Y$, Wang $L$, Zhang $M$, et al. Potential mechanism of interleukin-8 production from lung cancer cells: An involvement of EGF-EGFR-PI3K-Atk-Erk pathway. J Cell. Physiology. 2011; 227:35-43.

17. Chenzhi Jia, Wenchi Guan, Bing Li, et al. Effect of epidermal growth factor receptor inhibitor on acute respiratory inflammation induced by exogenous zinc ion in rats. J Journal of Zhengzhou University (Medical Edition). 2011; 46 (3):349-351.

18. Pan $\mathrm{H}-\mathrm{H}$, Hsiao Y-P, Chen P-J, et al. Epithelial growth factor receptor tyrosine kinase inhibitors alleviate house dust mite allergen Der p2-induced IL-6 and IL-8. Environmental Toxicology. 2019; 34(4): 1-10. 
19. Jing Xie, Rongli Wang. Effect of emodin on lung tissue inflammation and p38 MAPK expression in mice with pneumococcal pneumonia. Shandong Medical Journal. 2018; 58 (30):44-47.

20. Wang Q-W, Su Y, Sheng J-T, et al: Anti-influenza A virus activity of rhein through regulating oxidative stress, TLR4, Akt, MAPK, and NF-KB signal pathways. J PLOS ONE. 2018; 13(1): e0191793.

21. Cao J,Gong Y,Cai B, et al. Modulation of human bronchial epi-thelial cells by pneumococcal choline binding protein A, Humlmmunol. 2011; 72(1) : 37-46.

22. Li X, Zhou X, Li Y, et al. Lyn cooperating with lipid rafts regulates inflammatory responses in Klebsiella pneumoniae infection through the p38 / NF-KB pathway. J Eur J Immunol. 2014; 44:763773.

23. Choi YH, Jin GY, Piao HM, et al. Silibinin attenuates allergic airway inflammation in mice. J Biochem Biophys Res Commun. 2012; 427(3):450-455.

24. Hui Liu, Yongming Yao. Advances in the study of the interaction of intracellular inflammatory signaling pathways. Chinese Journal of Pathophysiology. 2005; 21(8),1607-1613, 1627.

25. Shengchun Zhao, Hongwei Chen, Guolin Wu, Chen Zhao. The association of NLRP3 and TNFRSF1A polymorphisms with risk of ankylosing spondylitis and treatment efficacy of etanercept. Journal of clinical laboratory analysis.2017; 31:e22138.

26. Zachary C. Hartman, Xiao-Yi Yang, Oliver Glass, et al. HER2 overexpression elicits a pro-inflammatory IL-6 autocrine signaling loop that is critical for tumorigenesis. Cancer Research. 2011;71(13):43804391.

27. Riley J.Morrow, Nima Etemadi, Belinda, et al. Challenging a Misnomer? The role of inflammatory pathways in inflammatory breast cancer. Mediators of Inflammation. 2017; 1-15.

28. H.Korkaya, G.I.Kim, A.Davis, et al. Activation of an IL-6 inflammatory loop mediates trastuzumab resistance in HER2+ breast cancer by expanding the cancer stem cell ell. J Molcel. 2012;47(4):570584.

29. R.Rodriguze-Barrueco, J.Yu,L.P.Saucedo-Cuevas, et al. Inhibition of the autocrine IL-6-JAK2-STAT3calprotectin axis as targeted therapy for HR-/HER2+ breast cancers. J Genes Development. 2015; 29(15):1631-1648.

\section{Table}

Table1. The reverse docking score of Rhein and related targets 


\begin{tabular}{cc}
\hline Targets & Rhein \\
\hline ERK1 & 8.476 \\
MEK1 & 5.643 \\
MP2K1 & 6.972 \\
EGFR & 6.032 \\
PAK1 & 8.012 \\
CREB1 & 8.785 \\
Grb2 & 7.085 \\
HRas & 7.506 \\
MyC & 6.446 \\
PDK1 & 7.408 \\
RasH & 8.015 \\
SRC & 8.254 \\
\hline
\end{tabular}

\section{Figures}

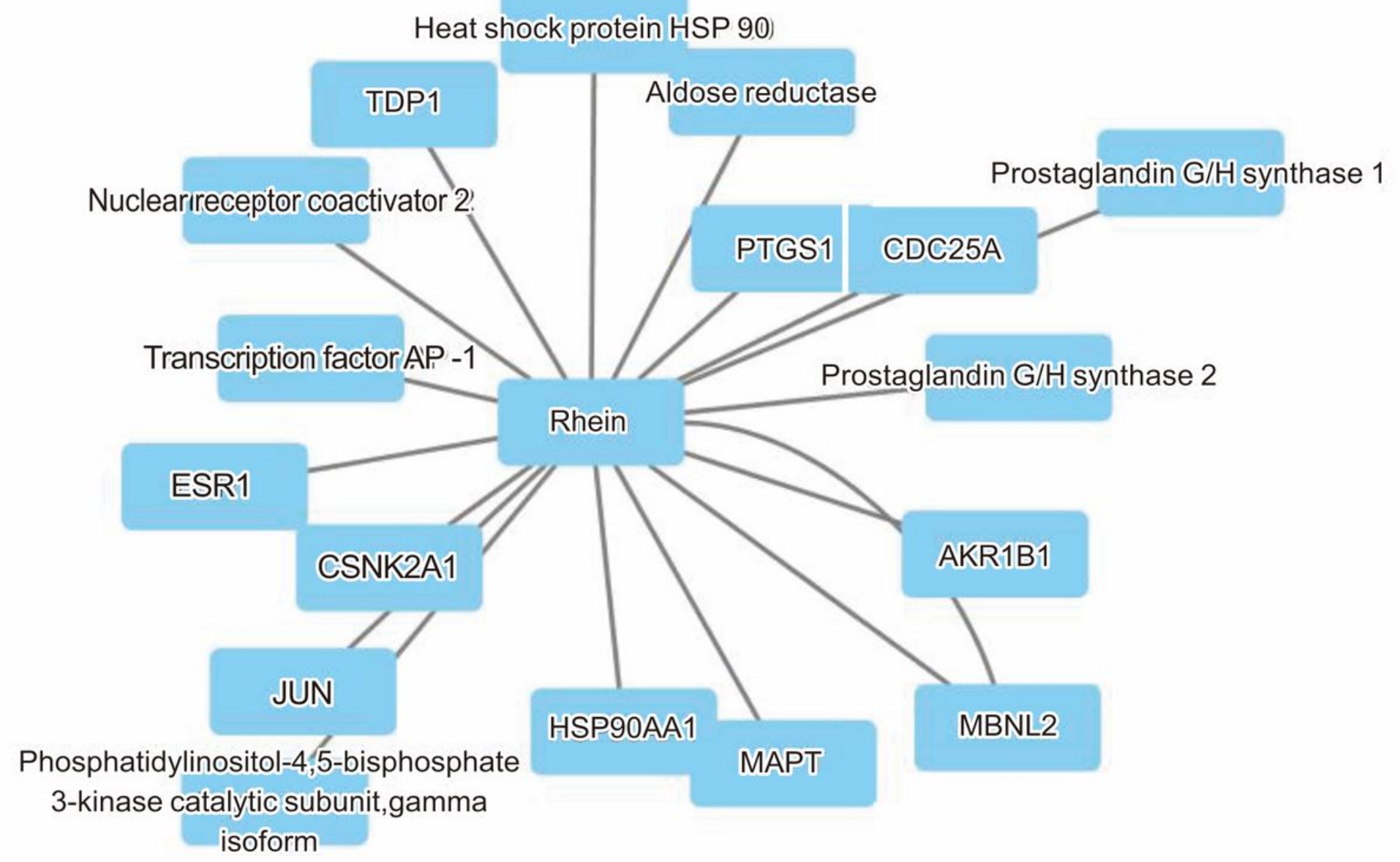

Figure 1

Seventeen predictive targets of Rhein. 


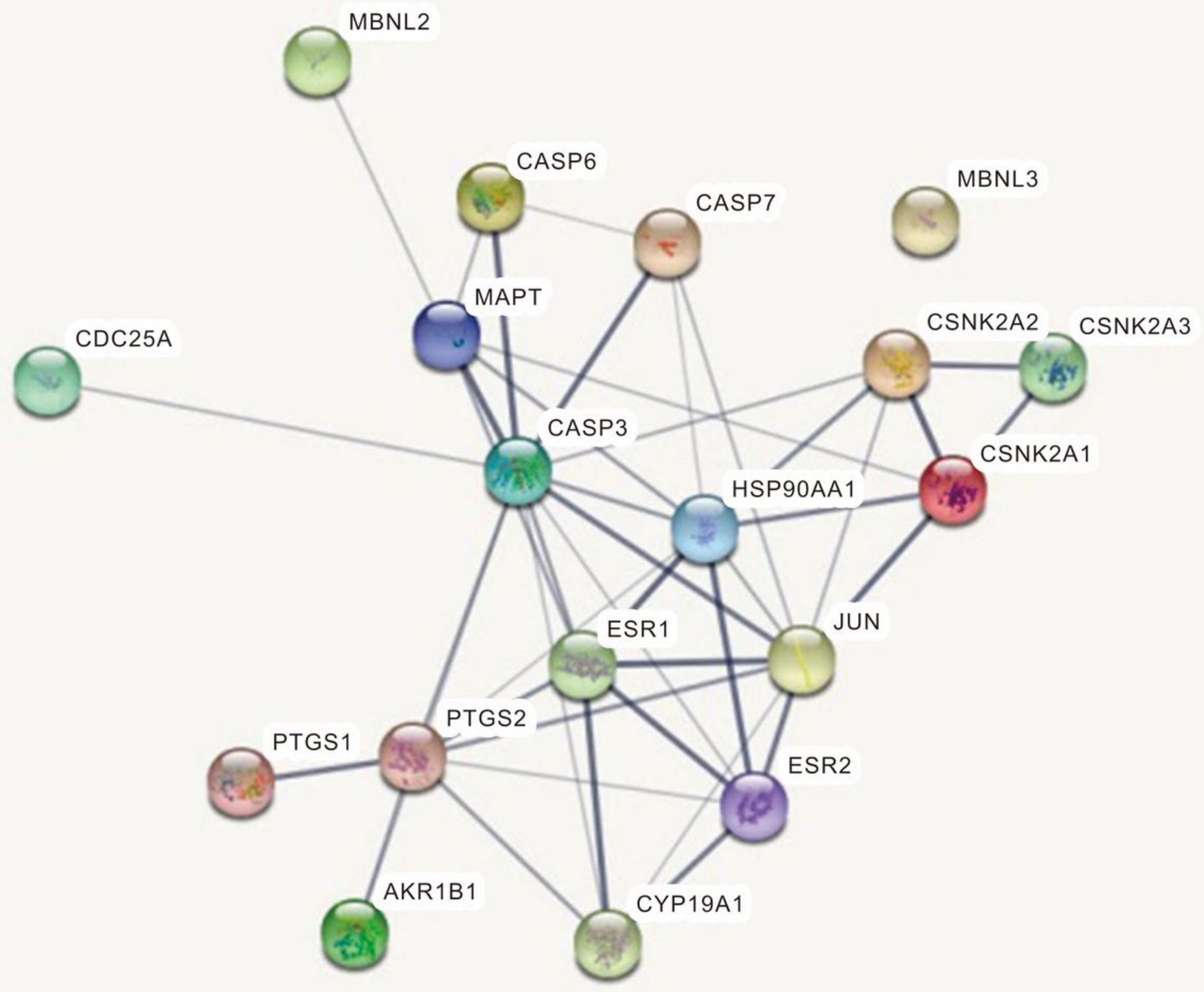

Figure 2

Network analysis map of interactions of Rhein target protein. 


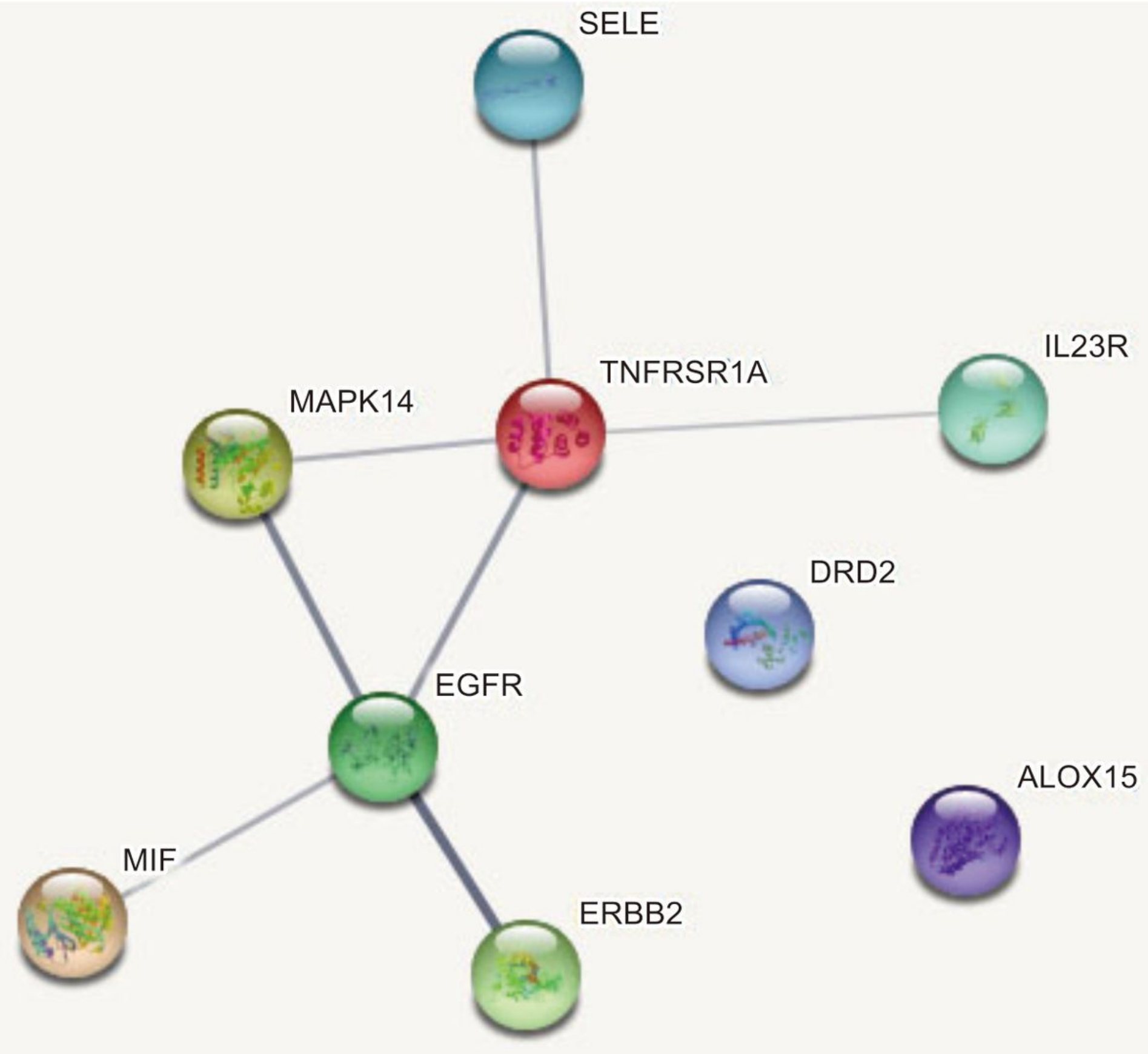

Figure 3

Seven proteins (SELE, MAPK14, TNFRSF1A, IL23R, EGFR, MIF, ERBB2) of nine proteins (SELE, MAPK14, TNFRSF1A, IL23R, EGFR, MIF, ERBB2, DRD2, ALOX15) interact with Rhein target protein. 


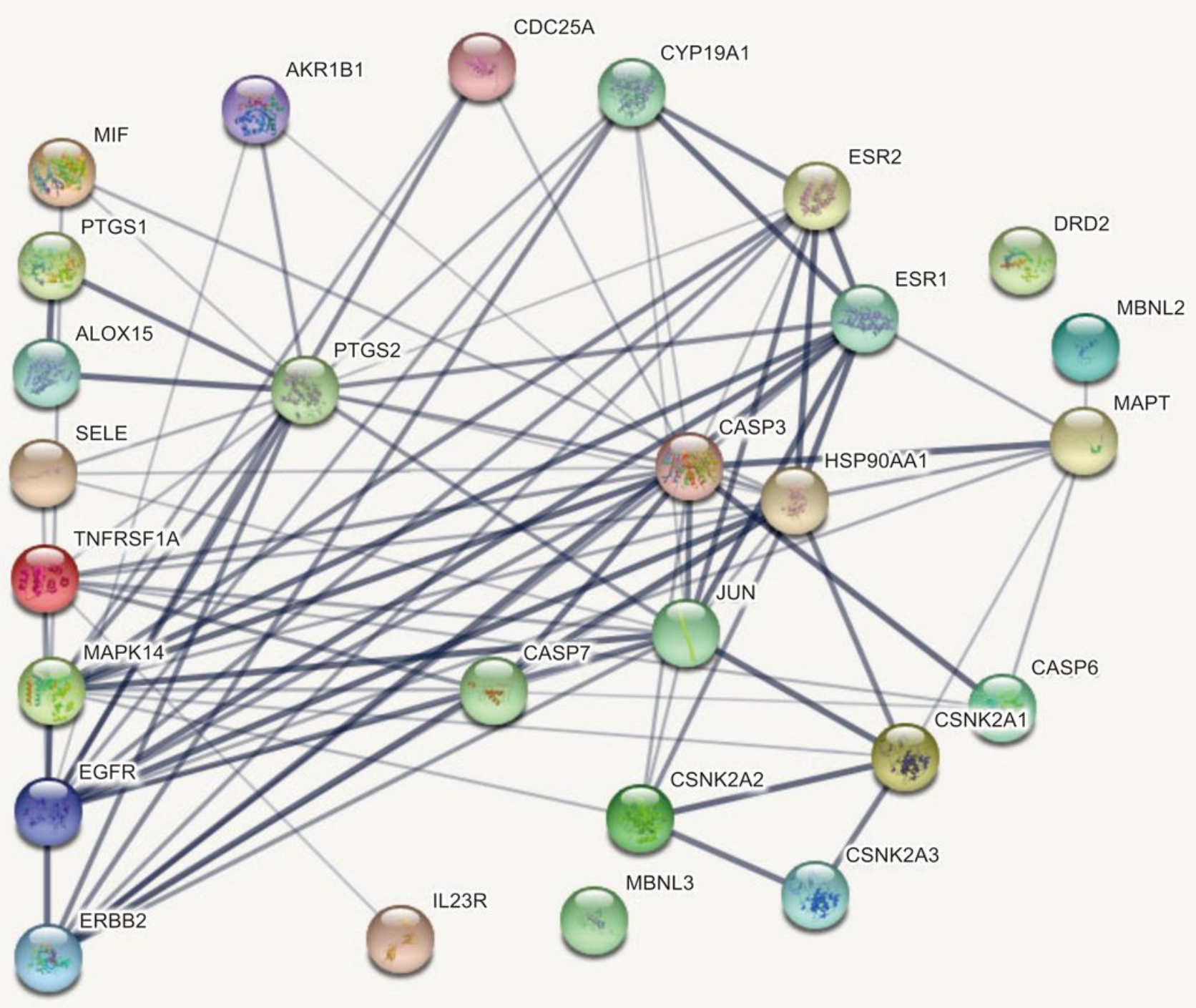

Figure 4

Anti-inflammatory targets of Rhein: MAPK14; ERBB2; TNFRSF1A; EGFR. 


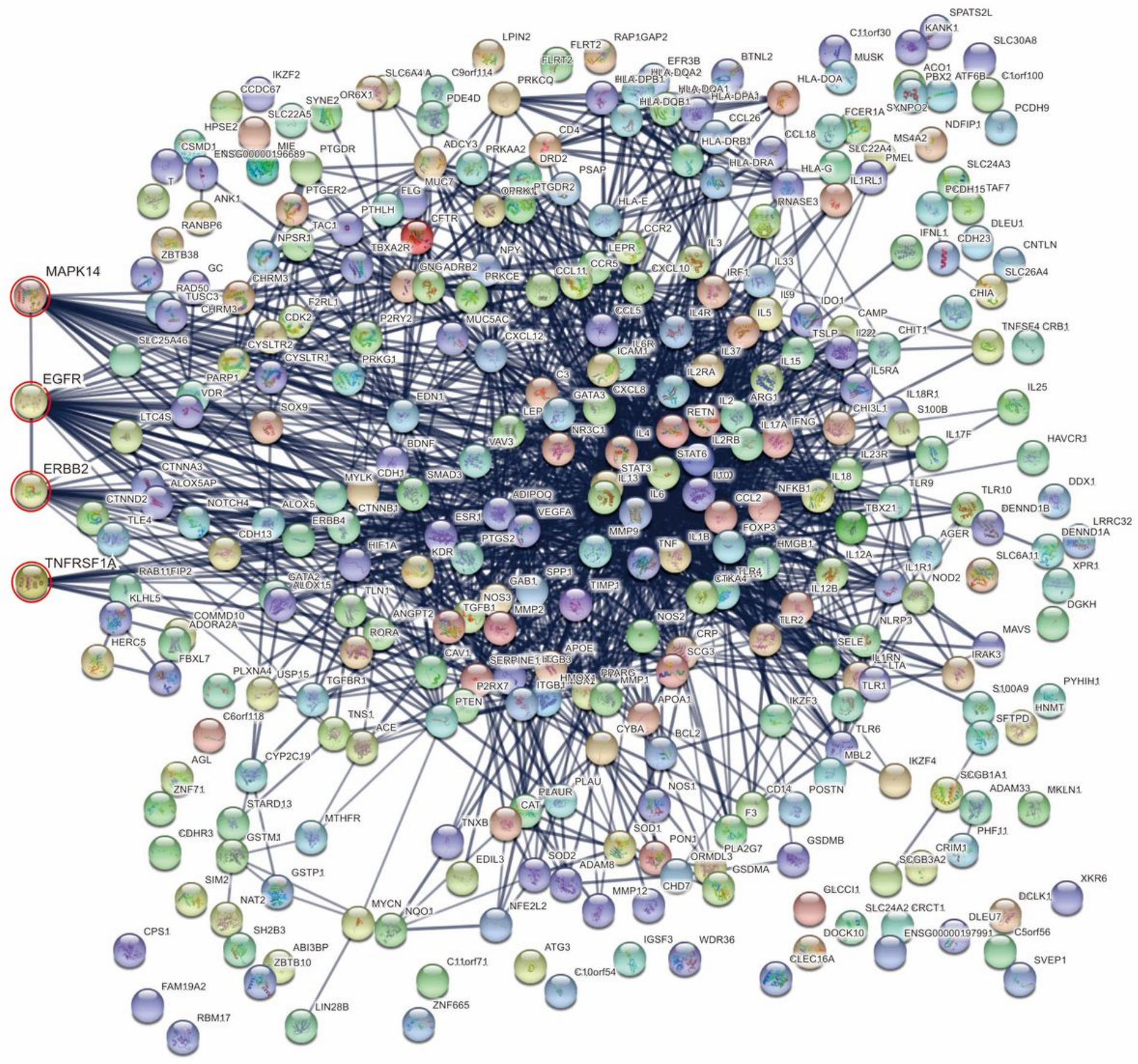

Figure 5

Four anti-inflammatory targets of Rhein related to asthma: MAPK14; EGFR; EERB2; TNFRSF1A. 


\section{Enriched Terms}

EGFR

ERBB2

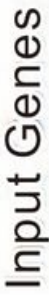

MAPK14
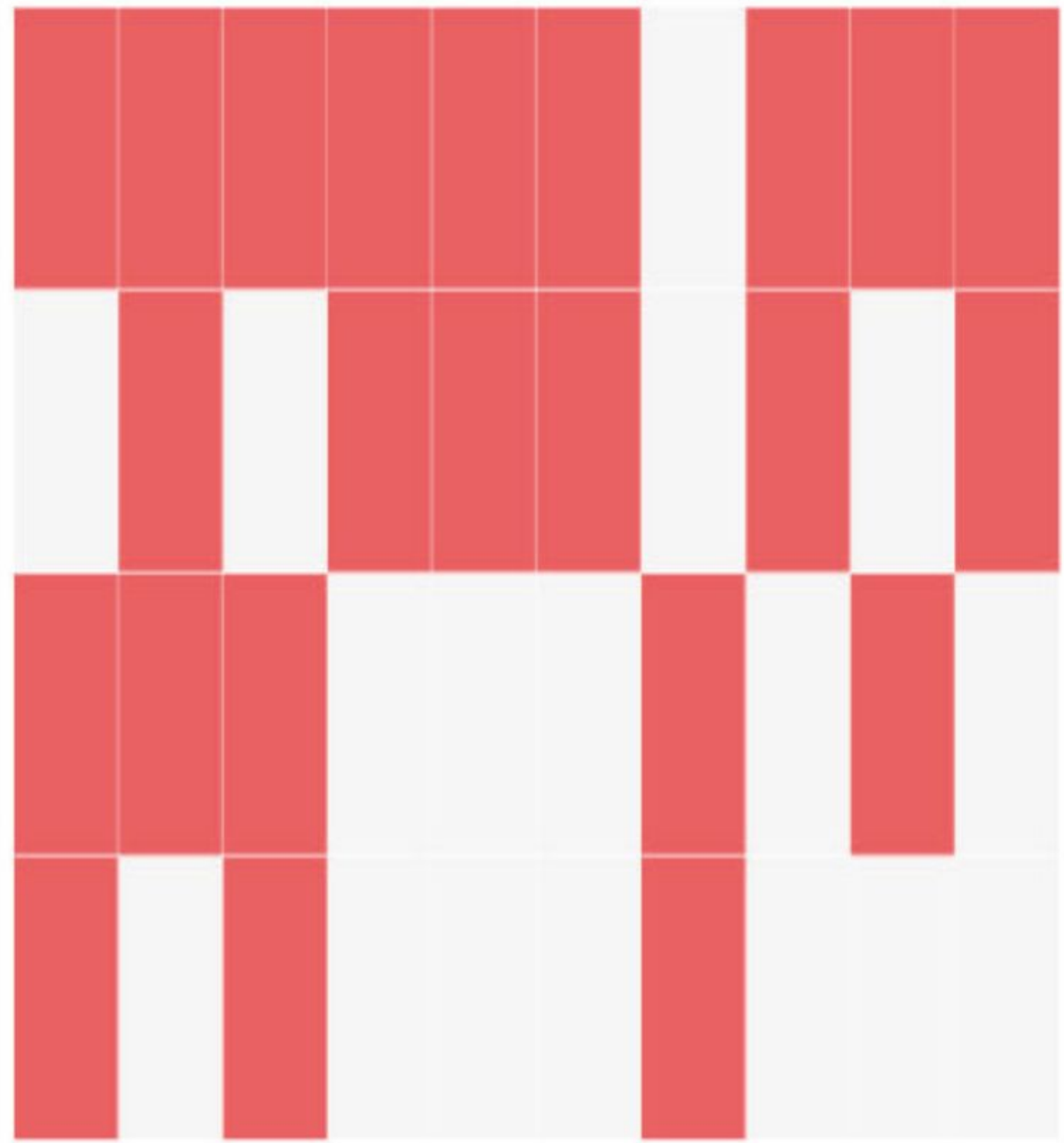

TNFRS.

\section{Figure 6}

Top ten signaling pathways among Anti-inflammatory target protein of Rhein related to asthma: MAPK signaling pathway; Hepatitis $\mathrm{C}$ signaling pathway; Epithelial cell signaling in Helicobacter pylori infection signaling pathway; Proteoglycans in cancer signaling pathway; Bladder cancer signaling pathway; Nonsmall cell lung cancer signaling pathway; Endometrial cancer signaling pathway; Pancreatic cancer signaling pathway; Central carbon metabolism in cancer signaling pathway; Amyotrophic lateral sclerosis (ALS) signaling pathway.

\section{Supplementary Files}


This is a list of supplementary files associated with this preprint. Click to download.

- SupplementaryTableSII.docx 